\title{
ON THE SINGLE-LINE SPECTRA OF MAGNESIUM AND OTHER METALS AND THEIR IONIZING POTENTIALS: *
}

\author{
BY \\ J. C. McLENNAN, F.R.S., \\ Director of the Physical Liboratory, University of Toronto, Toronto, Canadi.
}

\section{INTRODUCTION.}

It has been showi by Frank and Hertz ${ }^{1}$ that when heated mercury vapor is traversed by electrons possessing kinetic energy slightly above that acquired in a fall of potential of 4.9 volts the vapor is stimulated to the emission of the single spectral line $\lambda=2536.72$ A. U. It has also been shown by McLennan and Henderson $^{2}$ that a spectrum consisting of this single line only can be obtained from mercury vapor when it is bombarded by electrons possessing energy corresponding to aty fall of potential within a range beginning at about $j$ volts and extending up to slightly over ro volts. The investigation has also been extended by McLennan and Henderson to include a study of the radiation emitted by zinc and cadmium vapors when traversed by electrons. With these vapors they have found that single-line spectra can be obtained when the electrons traversing these vapors possess kinetic energy lying within a limited and clearly-defined range, which has not been fully investigated as yet, but which corresponds roughly to potential differences lying between 4 volts and I 3.6 volts. With zinc vapor the single-line spectrum consists of light of wave-length $\lambda=3075.99 \stackrel{\circ}{\AA}$. C., and with cadmium vapor of light of wave-length $\lambda=3260.17 \AA$. $\mathrm{C}$. It should also be pointed out here that the lines $\lambda=2536.72$ A. U., $\lambda=3075.99$ $\AA$. U., and $\lambda=3260 . \mathrm{I} 7 \stackrel{\circ}{\AA}$. U. are respectively the first members of Paschen's ${ }^{2 a}$ combination series $\nu=2, p_{2}-m, S$, for the clements, mercury, zinc, and cadmium.

* Presented at the meeting of the Section of Physics and Chemistry held Thursday evening, November II, IgI5.

${ }^{1}$ Verh. d. Deutsch. Phys. Ges., vol. il, p. si2 (IgI4).

"Proc. Roy. Soc. A., vol. 9I, D, 485 (I915).

2a Paschen, Ann, der Phys., No, Io, D. 869, I0I I. 


\section{THE SINGLE-LINE SPECTRUM OF MAGNESIUM.}

Since the publication of the results described above, the radiation from magnesium vapor traversed by electrons has been investigated by the writer, and it has been found with this element, too, that a single-line spectrum can be oltained if the electrons bombarding the vapor possess cnergy lying within a certain range whose limits also have not as yet been definitely determined, but which covers a portion at least of the ranges nentioned above for mercury, zinc, and cadmium. In carrying out these experiments the apparatus used and the procedure followed were precisely the same as those described in the paper by McLennan and Henderson. The form of arc used is that shown in Fig. I. The apparatus consisted of a tube of fused quartz possessing

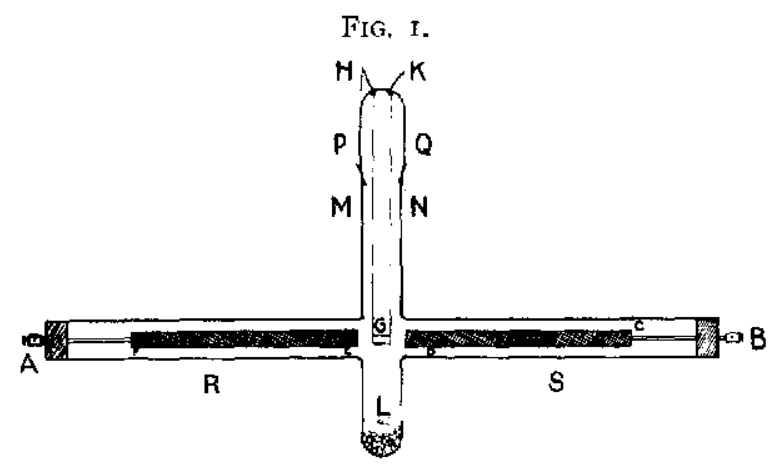

three arms, $R, S$, and $M N$, and a receptacle $L$. Some of the metal to be used in the arc was placed in the receptacle $L$, and two rods of the same metal, $F E$ and $D C$, werc attached to two wires, and these latter were in turn fastened to two brass plugs, $A$ and $B$, which were sealed into the tubes $R$ and $S$ with mastic wax. A snall piece of sheet platinum was attached to two wires which constituted the heating circuit, and these were sealed with platinum wire into a glass tube $P Q$ at $H$ and $K$. The open end of the glass tube $P Q$ was ground so as to fit exactly into the end of the quartz tube $M N$, as shown in the diagram. The arms $M N, R$, and $S$ were each about $40 \mathrm{~cm}$. long and it was found with this length that when the receptacle $L$ was strongly heated with a Bunsen burner the wax joints at $A$ and $B$ and the ground one at the end of the tube $M N$ remained quite cool. In the experiments the plate $G$ was coated with a thin layer of either cal- 
cimm oxide or barium oxicle. When the tube was in operation the terminals of an auxiliary heating circuit were attached at $H$ and $K, B$ and $K$ were joined by a wire, and the arcing voltage was applied between $B$ and $A$, the latter being the positive terminal. With this arrangenent $G$ and $D$ constituted a double cathode. The tube was highly exhausted with a Gaede mercury pump through a glass tube which was sealed into an opening in the brass end-piece at $A$.

In taking photographs the plate $G$ was brought to incandescence by means of the atriliary heating current, the metal in $L$ was strongly heated with the hame of a Bunsen burner so as to keep the plate $G$ surrounded with the vapor of the metal, and the collimator of a small spectrograph with a quartz train was directed at the incandescent plate $G$. A short tube of asbestos was

FIG. 2.

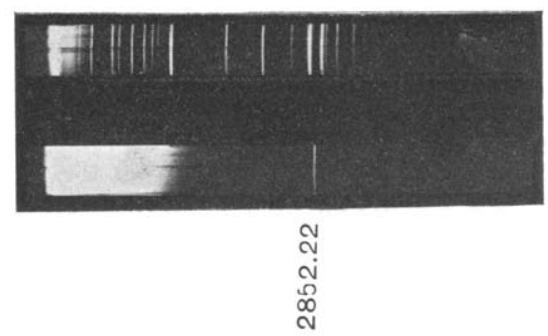

attached to the quartz tube directly in front of this plate so that the radiation from the arc passed through it to the slit of the spectroscope. This arrangement was found necessary in order to cut off the radiation from the Bunsen flame itself. It should be noted that in studying the radiation from mercury vapor the electrodes $C D$ and $F E$ were simply stout iron wires.

With the arrangement just described it was found that when

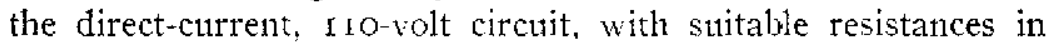
series, was applied to the terminals $A$ and $B$, and the plate $G$ brought to incandescence, strong ares could be maintained for hours with mercury, zinc, or cadmium. With the 220 -volt circuit applied the arcs of all three metals could be made most intense, and could also be maintained for long periods. With the 220 -volt circuit it was found that, when the arc was once struck, it could be casily maititained for a consiclerable time without the continued use 
of the oxy-cathode $G$. With low voltages, however, it was always necessary to maintain the plate $G$ at incandescence in order to keep the arc established.

With magnesium the arcs could be maintained for short periods only and it was difficult to keep the vapor of the metal from condensing on the walls of the tube. The single-line spectrum of this

FIG. 3.

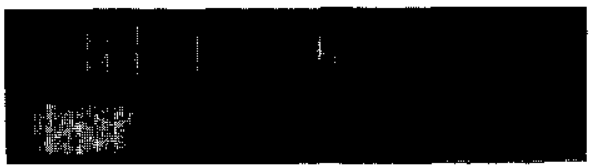

$N$
$\stackrel{0}{*}$
$\stackrel{0}{N}$

element consists of light of wave-length $\lambda=2852.22 \stackrel{\circ}{\mathrm{A}}$. U. The ordinary spark spectrum of magnesium in air is shown in the upper row in Fig. 2, and the single-line spectrum of the vapor of the metal in the second row of the same figure. The latter was obtained with a three-hour exposure, and the electrons which stimtilated the vapor to the emission of this radiation acquired their kinetic energy

FIG. 4.

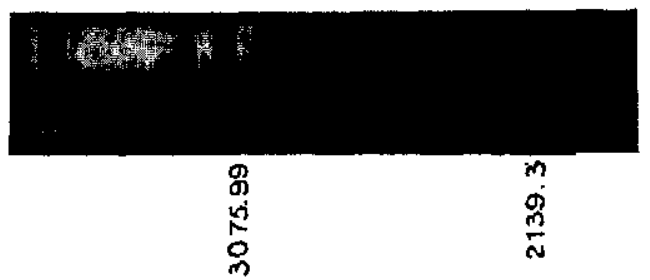

with an arcing potential of 8.2 volts applied between the Wehnelt cathode and the positive terminal.

The single-line spectrum for mercury vapor which is shown in Fig. 3, at $\lambda=2536.72 \AA$. U., was obtained with an arcing potential difference of 9 volts with an exposure of two hours and a half. This single-line spectrum was also obtained with a potential fall of 5 volts, but it was only just visible on the plate with a five-hour exposure. With 3 volts and a five-hour exposure the line was not obtained. The line $\lambda=3075.99 \AA$. U., shown in 
the lower spectrogram of Fig. 4, was obtained with zinc vapor and an arcing potential of 10.5 volts with an exposure of three hours, and the line $\lambda=3260.17 \AA$. U, shown in the lower spectro-

FIG. 5.

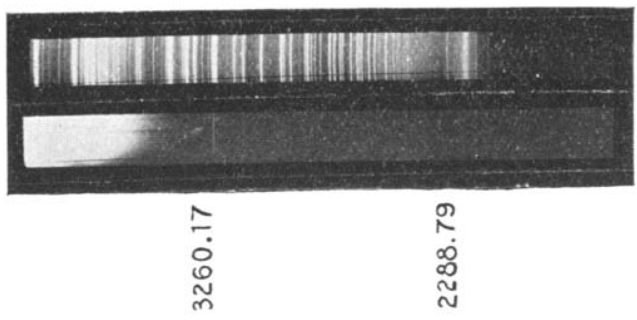

gram of Fig. 5, was obtained with cadmium vapor with an arcing potential difference of 13.6 volts. With an arcing potential of 3.4 volts and a three-hour exposure no trace of the line was obtained with this element.

\section{THE ABSORPTION SPECTRA OF MAGNESIUM AND OTHER METALLIC VAPORS.}

In a paper recently published by McI.ennan and Edwards ${ }^{3}$ it has been shown that in the absorption spectrum of mercury there is an absorption band at $\lambda=2536.72 \AA$. U., and one at $\lambda=1849.6 \AA$. U. With this vapor it has been found that there is also a complex band obtainable at $\lambda=2338 \mathrm{~A}$. U., when high vapor densities are used. With zinc and cadmium vapors it has been shown by the same writers that the absorption spectra consist of but two absorption bands. With zinc vapor these are at $\lambda=3075.99 \AA . \mathrm{U}$., and at $\lambda=2 \mathrm{r} 39.3 \AA$. U., and with cadmium vapor they are at $\lambda=3260 . \mathrm{I} \gamma \AA$. U., and $\lambda=2288.79 \AA$. U. As pointed out above, the lines $\lambda=2536.72 \AA$. U, $\lambda=3075.99 \AA$. U., and $\lambda=3260$. I $7 \stackrel{\circ}{\Lambda}$. U. are the first members of Paschen's combination series for the three elements represented by $\nu=2, p_{2}-m$, $S$, and they are therefore the lines of this series corresponding to the value $m=\mathrm{J} .5$. Again, it will be seen, by referring to

${ }^{3}$ McLennan and Edwards, Froc. Roy. Soc. of Canada, 1915; Phil. Mag., November, 195 . 
Paschen's ${ }^{t}$ paper, that the lines $\lambda=\mathrm{J} 849.6 \mathrm{~A}$. U., $\lambda=2$ เ $39 . \mathrm{I} 3$ A. L., and $\lambda=2288.79 \AA$. L. are the first members of the series $\nu=\mathrm{r} .5, \mathrm{~S}-m, P$, predicted by Paschen and later identified in part by Wolff: for the three elements mercury, zinc, and carlmiun.

It does not appear, from communications which have cone to the notice of the writer, that a series corresponding to $\nu=1.5$, $S-m, P$ has as yet been identified in the spectrum of magnesium, but if we assume that the line $\lambda=2852.22 \mathrm{~A}$. U. is the first line in the combination series $v=2, p_{2}-m, S$ for this element, sufficient information is given in a paper by Dunz ${ }^{6}$ to calculate the first and the last member of the series $\nu=1.5, S-m, P$ for this metal. In the paper by Dunz referted to, the frequency of $\nu=2, p_{2}$ in the magnesium spectrum is given as $39,793.2 \mathrm{I}$. If we take the frequency of the line $\lambda=2852.22 \mathrm{~A}$. $\mathrm{L}$. to be $35,050.45$, it follows that the frequency $\nu=\pi .5, S$ is equal to $74,843.66$. This will then be the frequency of the last line of the series spectrum of magnesium given by $\nu=\mathrm{I} .5, S-m, P$. Again, in the paper by Dunz the frequency $\nu=2, P$ is given as $26,612.7$, and from this it follows that the frequency of the frst line in the series $\nu=1.5$, $S-m, P$ (i.e., $\nu=$ T.5, $S-2, P$ ) is $48,230.96$. "lhis, it will be seen, is the frequency of the line $\lambda=2073.36 \AA$. L. If then the vapor of magnesium were to act, as regards absorption, in a manner analogous to the vapors of mercury, zinc, and cadmium, the absorption spectrum of magnesium vapor should contain absorption bands at $\lambda=2852.22 \AA$. L. and at $\lambda=2073.36 \stackrel{\circ}{\AA} \mathrm{L}$. On looking up the literature on the subject it was found that Wood and Guthrie ${ }^{7}$ and Eder and Valenta ${ }^{8}$ had already shown that there is an absorption band in this spectrim at $\lambda=2852.22 \stackrel{\circ}{\mathrm{A}}$. $\mathrm{U}$., but, as no one seemed to have found any band at $\lambda=2073.36 \AA$. U., some experiments were made to see if it really existed. The experiments confirmed its existence, and a reproduction of one of

* Paschen, loc. cit.

${ }^{5}$ Wolff, Ann. der Phys., Igr3, vol. xlii, D. 525.

"D:1nz, "Bearbeitung unserer Kenntnisse von den Serien," Inaug. Diss., Tübingen, I9I I.

"Wood and Guthrie, Astrophys. Jour., vol. xxix, No: I, p. 21 I (Igog).

"Eder and Valenta, "Atla; Typischer spectren," Table xxvii. 
the photographs taken is shown in Fig. 6. The upper portion of this figure was taken with the light from the spark between magnesium terminals in air, and the lower onc with the same light after it had traversed an evacuated clear fused quartz tube containing heated non-luminous magnesium vapor. As the reproduction shows, absorption occurred at $\lambda=28_{52.22} \AA$. $\mathrm{C}$. and at $\lambda=2073.3^{6} \mathrm{~A}$. $\mathrm{U}$., as well. In addition, i narrow absorption band appears at $\lambda=2536.72 \therefore$. C. This band also appeared in the experiments of Mclennan and Edwards referred to above in the absorption spectrum of zinc and cadmium vapors, and it was no doubt due to a trace of mercury vapor which may have come from mercury originally present as an impurity in the metals or from mercury which got into the absorption tubes containing the vapors

FIG. 6.

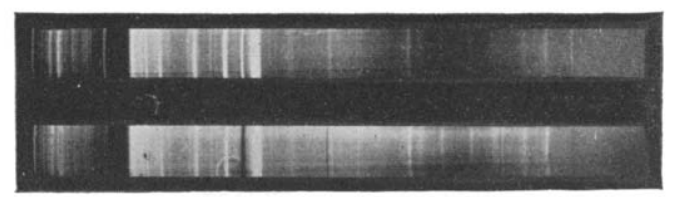

FIG. 7.

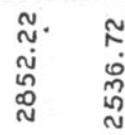

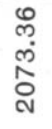

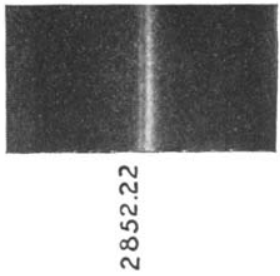

when these tubes were exhausted by the Gaede mercury pump. From this result it will be seen that the absorption spectrum of magnesium vapor is exactly analogous to the absorption spectra of mercury, zinc, and cadmium. The analogy, moreover, between the absorption spectrum of magnesium and that of mercury is more perfect than would appear from the above considerations, for the absorption band at $\lambda=2536.72 \AA . \mathrm{L}$., in the absorption spectrum of mercury vapor, comes out with small vapor densities as two narrow absorption bands whose wave-lengths have been given by $R$. W. Wood ${ }^{9}$ as $\lambda=2536 \AA$. U. and $\lambda=2539$ A. U. The absorption band at $\lambda=2852.22 ~ A . ~ U$., in the absorption spectrum of magnesium vapor, has also been found to consist of two narrow, sharply-defined bands very close together. These are shown in the reproduction in Fig. 7 , which was obtained by

${ }^{3}$ R. W. Wood, Astrophys. Jour., vol. xxvi, No. I, p. 41. 
greatly enlarging a negative of the band in Fig. 6 at $\lambda=2852.22$ A. U. The bands at $\lambda=3075.99 \mathrm{~A}$. L. and $\lambda=3260$. I 7 A. L., in the absorption spectra of zinc and cadmitum vapors, have not as yet been resolved into analogous doublets.

\section{THE SINGLE-LINE AND ABSORPTION SPECTRA OF CALCIUM, STRONTIUM, AND BARIUM VAPORS.}

The attention of the writer was recently directed to papers by Charles De Watteville ${ }^{10}$ on " Flame Spectra." In these papers the author has pointed out that the spectrum of the radiation from the cone of a flame fed by the spray of salt solutions of a number of elements consists in some cases of but a single strong line, and in others of a single strong line accompanied by a number of very much fainter ones.

These strong lines for the different metals are:

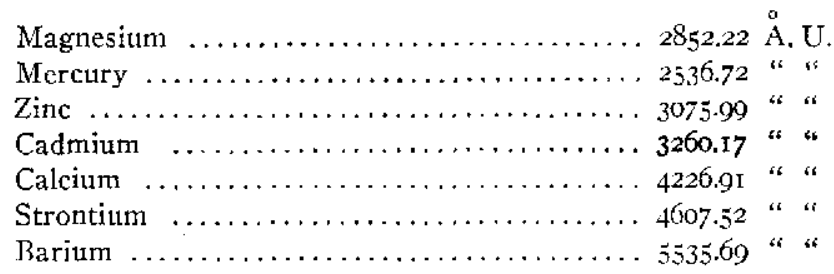

De Watteville in his papers points out that these metals all belong to Mendelejeff's second group, and he also presents a number of considerations which led him to conclude that these lines are corresponding ones in the spectra of these seven metals. If we accept this conclusion it follows, from what goes before, that calcium, strontium, and barium vapors should emit singleline spectra of the wave-lengths just mentioned if bombarded by electrons possessing the requisite amount of energy. It also follows that absorption bands should appear in the absorption spectra of the vapors of these metals at the respective wavelengths given above. The only in formation regarding the absorption spectra of these metals which the writer has been able to find is contained in the "Atlas of Spectra," by Eder and Valenta. ${ }^{11}$ In Table XXIT of this atlas a spectrogram is given of the arc spectrum of calcium which shows a strong reversal band

${ }^{10}$ De Watteville, Phil. Trans, Roy. Soc., Series A, vol. 204, pp. 139-168 (1904), and C. R. No. 142, I906.

iz Eder and Valenta, loc. cit. 
at $\lambda=4226.9 \mathrm{I} A$. $\mathrm{L}$, but it also shows in addition faint re-

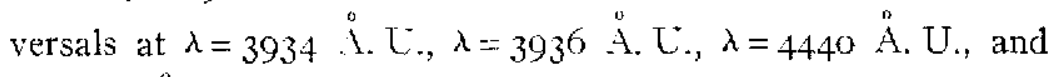
$\lambda=4460 \AA$. $\mathrm{C}$. In the same table a spectrogram of the arc spectrum of strontium bromide also shows reversals at $\lambda=4607.52$ A. U. and $\lambda=4078 \AA$. U., and in Table XXIII a spectrogram of the arc spectrum of barium bromide shows reversals at $\lambda=$ $5535.69 \AA$. U. and $\lambda=4554 \AA$. $\mathrm{C}$. No direct investigation of the absorption spectrum of the vapors of these metals appears to have been made as yet, but experiments are now being made by the writer, and it is expected that some information on this subject will be soon forthcoming.

If the line spectra of calcium, strontium, and barium be analogous to those of mercury, zinc, cadmitum, and magnesium, then the spectra of the first-named metals should each contain a series given by $\nu=1.5, S-m, P$. Assuming that the lines $\lambda=4226.9 \mathrm{I}$ A. U., $\lambda=4607.52 \AA$. U., and $\lambda=5535.69 \AA$. U. are the first members of the series $\nu=2, p_{2} \cdots m, S$, for calcium, strontium, and barium (i.e., that they are given by $\nu=2, p_{2}-\tau .5, S$ ), it follows that if $\nu=2, p_{2}$ be known for the spectra of these elements then the frequencies represented by $\nu=1.5 . S$ can be calculated for them, and these would give the waves of shortest length in the series $\nu=1.5, S-m, P$. Firther, a knowledge of the values of $\nu=2, P$ for the spectra of the three elements would then give the first members of the same three series.

From the paper by Dunz it will be seen that for the spectra of calcium and strontium the values $\nu=2, p_{2}$ are respectively $34,-$ 089.47 and $3 \mathrm{I}, 420.38$. Taking the frequencies of the lines represented by $\nu=2, p_{2}-1.5, S$ for calcium and strontium to be $23_{1}-$ 644.25 and $21,705.82$, it follows that the frequencies of $y=1.5, S$ for these two elements are respectively $57,733.72$ and $53,126.2$. These frequencies correspond to $\lambda=1732.1 \AA$. U. and $\lambda=1882.3$ $\AA$. U., and these wave-lengths, therefore, should be the limiting ones in the series $\nu=1.5, S-2, P$ for the two elements mentioned. Again, the frequencies $\nu=2, P^{x}$ for calcium and strontium are given in the paper by Dunz ${ }^{12}$ as 27.5 10.2 and 25.745.2, so that,

${ }^{12}$ Dunz, loc cit. 
combining these with the values of $\nu=$ T.5. S griven above, we have $30,223.52$ and $27,380.9$ as the frequencies represented by $\nu=1.5$, $S-2, P$ in the spectra of calcium and strontium. As the wavelengths corresponding to these frequencies are $\lambda=3308.6 \AA$. $\mathrm{L}$. and $\lambda=3652.2 \mathrm{~A}$. U., it would follow that these wave-lengths are respectively the first members of the series $\nu=1.5, S-m, P$ in the spectra of calcium and strontium. The paper by Dunz does not appear to contain data which would enable one to determine the frequency $\nu=2, P$ for barium. However, it gives the frequency $\nu=2, p_{2}$ as $29,35^{\circ} .4$ for this element. Taking then the wave-length $\lambda=5525.69 \AA$. $\mathrm{L}$. as the one whose frequency is given by $\nu=2, p_{2}-1.5, S$ in the spectrum of barium, it follows that for this element the frequency $\nu=$ I.5, $S$ is equal to 47 ,414.99. As this is the frequency of $\lambda=2109.04 \mathrm{~A}$. U., it would appear that for barium this wave-length is the shortest one in the $\nu=\mathrm{I} .5, S-m, P$ series. From the considerations presented it would appear, therefore, that for calcium, strontium, and barium $\lambda=4226.9 \mathrm{I} \stackrel{\circ}{\AA}$. U., $\lambda=4607.52 \AA$. $\mathrm{L}$, and $\lambda=5535.69 \AA$. U. are respectively the first members of the series $\nu=2, p_{2} \cdots, n$, and that for the same elements $\lambda=1732 . \mathrm{I} \stackrel{\mathrm{A}}{\mathrm{C}} \mathrm{L} ., \lambda=\mathrm{I} 882.3 \mathrm{~A}$. $\mathrm{L}$., and $\lambda=2 \mathrm{I} 09.4 \stackrel{\AA}{\mathrm{A}}$. $\mathrm{C}$. are respectively the last members of the series represented by $\nu=1.5, S-m, P$. In addition, the first member of the series $\nu=1.5, S-m, P$, in the spectrum of calcium should be $\lambda=3308.6 \AA$ U., and the first member of the same series for strontium, $\lambda=3652.2 \AA$. L. The information regarding the details of the series spectra of barium at present available is not sufficient, apparently, to enable one to calculate the wavelength of the first member of the series $\nu=\mathrm{I} .5, S-m, P$ for this element.

As the absorption spectra of the vapors of mercury, zinc, cadmium, and magnesium have absorption bands at the two wavelengths given by $\nu=2, p_{2}-1.5, S$ and $\nu=\mathrm{I} .5, S-2, P$, it would follow, from the arguments presented, that if the vapors of calcium, strontitum, and barium behave in an analogous manner there should be absorption bands at $\lambda=4226.9 \mathrm{I} \AA$. $U$. and $\lambda=3308.6$ 
$\AA$. U. in the absorption spectrum of calcium vapor, and also bands at $\lambda=4607.52 \AA$. $L$. and $\lambda=3652.2 \AA$. $L$. in the absorption spectrum of strontium vapor. The absorption spectrum of the vapor of barium should contain a lyand at $\lambda=5535.69 \AA$. U., but, as previously stated, the information available is not sufficient to predict the position of the second absorption luand.

As already pointed out above. we have sufficient information available already regarding the absorbing properties of calcium, strontium, and barium vapors to confirm in part the findings just presented, but to confirm them fully additional experiments will have to be made.

\section{THE SINGLE-LINE AND ABSORPTION SPECTRA OF THALLIUM.}

Ramage, ${ }^{13}$ in a paper on the comparative study of the spectra of some of the elements, points out that the flame spectrum of thallium also consists of the single line $\lambda=5350.65 \AA$. L. If we assume, as we have done in dealing with the single-line flame spectra of calcium, strontium, and barium, that the frequency of the line $\lambda=5350.65 \AA$. $\mathrm{C}$. is that of the first line in the series $\nu=2$, $p_{2} \cdots m, S$ for the spectrum of thallium (i.e., that it is given by $\left.\nu=2, p_{2}-1.5, S\right)$, then, since for this element Dunz gives the frequency $\nu=2, p_{2}$ as 49.262 .55 , it follows that the frequency $\nu=1.5$, $S$ for the spectrum of thallium is equal to $67,946.77$. This frequency corresponds to the wave-length $\gamma=\mathrm{I} 47 \mathrm{I} .74 \stackrel{\mathrm{A}}{\mathrm{A}}$. U., and this wave-length should, therefore, be the last one in the series $\forall=1.5, S-m, P^{\prime \prime}$ of the spectrum of the element thallium.

As the frequency $\nu=2 . P$ is not known for the spectrum of thallium, one is not able as yet to calculate the frequency $\nu=\mathrm{T} .5$, $S-2, P$ (i.e., the frequency of the first line in the series $\nu=\mathrm{r} .5$, $S-m, P)$.

If we assume the behavior of thallium vapor as regards absorption to be the same as that of the vapor of mercury, zinc, cadmium, and magnesium, we should expect to find absorption bands in its absorption spectrunl at the wave-lengths given by the frequencies $\nu=2, p_{2}-1.5, S$ and $\nu=1.5, S-2, \Gamma$.

${ }^{1 .}$ H. Ramage, Proc. Roy. Soc, vol. 70, p. I (Igo7).

VoL. I8I, No. 1082-15 
Wood and Guthric, in their paper on the absorption spectra of metallic vapors, state that with thallium they found a strong absorption band at $\lambda=2380 \AA$. U., and fainter ones at $\lambda 3230 \AA$. U., $\lambda=3092 \AA$. L., and $\lambda=2530 \AA$. U., but they do not seem to have found any indication of absorption at $\lambda=5350.65 \AA$. U., which is the wave-length whose frequency is given by $\nu=2, p_{2}-1.5, S$. It is just possible that it would require high vapor densities and high resolving power to bring out this band, for in the case of zinc vapor it was found necessary to work under these conditions to bring out the absorption band at $\lambda=3075.99 \AA$. U.

\section{THE IONIZING POTENTIALS OF DIFFERENT ELEMENTS,}

In the paper by McLennan and Henderson attention was drawn to a paper by Frank and Hert ${ }^{14}$ which described experiments leading to the conclusion that the minimum energy required to ionize an atom of mercury was that acquired by an electron in passing through a fall of potential of 4.9 volts. Attention was also drawn in this paper to a second communication by Frank and Hert $^{15}$ in which it was shown that in the quantum relation $V e=h \nu$ where $h=6.6 \times$ Io $^{-27}$ erg sec. 4.9 volts is the potential fall which corresponds to the frequency of the line $\lambda=2536.72$ $\AA$. U. From this it follows that for mercury atoms at least a knowledge of the wave-length of the single line characterizing the single-line spectrum of this element is sufficient to enable one to calculate the ionizing potential. If the relation just pointed out be applicable generally to all the elements, it follows that if the vapor of an element can be shown to be capable of exhibiting a single-line spectrum the frequency of this single spectral line may be used to deduce the minimum amount of energy required to ionize the atoms of that element. From the considerations already presented in this paper it will be seen that the wavelengths of the single spectral lines in the single-line spectra of the elements have the frequencies given by $\nu=2, p_{2}-1.5, S$, and, as these frequencies are known for mercury, zinc, cadmium,

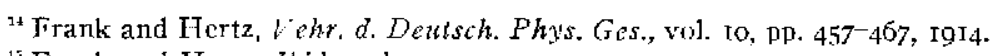

${ }^{15}$ Frank and Hertz, loid., vol. II, P. 512, I9I4. 
magnesium, and probably also for calcium, strontium, barium, and thallium, it follows that if Frank and Hertz have put the correct interpretation upon their experiments-and it may be added here that their experiments have apparently been confirmed quite recently by Newman ${ }^{16}$ - then the ionizing potentials for the atoms of all these elements can be calculated by the relation $V e=h \nu$. The resilts of this calculation are given in Table 1 .

TABLE I.

\begin{tabular}{|c|c|c|c|}
\hline Element & $\begin{array}{c}\text { Wave-length with } \\
\text { irequency } \\
v=2, t_{2}-1.5, S\end{array}$ & \multicolumn{2}{|c|}{$\begin{array}{l}\text { Ionizing potential } \\
\text { calculated on basis } \\
\text { of conclusions of } \\
\text { Frank and Hertz }\end{array}$} \\
\hline Mercury & $2536.72 \mathrm{~A} . \mathrm{U}$. & 4.9 & volts \\
\hline Zinc $\ldots$ & $.3075 .99 " "$ & 3.96 & " \\
\hline Cadmium . & . $3260.17 " “$ & 3.74 & “ \\
\hline Magnesium & . . 2852.22 & 4.28 & $"$ \\
\hline Calcinm .. & $42260 \mathrm{gr}$ " " & 2.89 & $" 4$ \\
\hline Stronlium & 4607.5214 & 2.65 & $"$ \\
\hline Barium ... & " & 2.24 & $"$ \\
\hline Thallium . & 5350.65 " & 2.28 & $a$ \\
\hline
\end{tabular}

In the paper by McLennan and Henderson it was pointed out that in order to obtain the single-line spectra with mercury, zinc, and cadmium vapors it was necessary that the electrons bombarding these vapors should not possess kinetic energy greater than that acquired in passing through falls of potential of 12.5 volts, I I.8 volts, and I 5.3 volts respectively for the three vapors. If the electrons possessed kinetic energy greater than that given by these voltages, visible arcs were struck and the many-lined spectra were obtained for the three elements. In the paper mentioned it was also stated that as these voltages gave with the relation $V \boldsymbol{e}=h \nu$ approximately the wave-lengths of the limiting lines in the series $\nu=1.5, S-m, P$ for the three elements, the results might be interpreted as indicating possibly a second type of ionization which the atoms of these elements might be capable of undergoing. If this interpretation should turn out to be correct, it would follow, since the frequency of the liniting lines in the series $\nu=1.5 . S-m, P$ are given by $\nu=1.5 . S$, that the ionizing potentials of the second type are given by $V=h \times$ (I.5, $S) / e$. Applying this relation to the restlts already obtained and

${ }^{16}$ Newman, Phil. Mag., 28, pp. 753-756, November, Ig14. 
given above, the ionizing potentials of the second type have been calculated for mercury, zinc, and cadminm, magnesium, calcium, strontium, barium, and thallium, and are given below in Table II.

\begin{tabular}{|c|c|c|c|c|}
\hline \multirow{2}{*}{$\begin{array}{l}\text { Element } \\
\text { Mercury }\end{array}$} & \multicolumn{2}{|c|}{$\begin{array}{c}\text { Wave-length cor:esyonding } \\
\text { tofrequency } \\
y=1.5, S\end{array}$} & \multicolumn{2}{|c|}{$\begin{array}{c}\text { Ionizing potentials } \\
\text { calculated from } \\
V=h \times(\mathrm{x}, 5, S) / e\end{array}$} \\
\hline & $\ldots$ I 188.0 & A. L. & 10.27 & volts \\
\hline Zinc $\ldots .$. & $\ldots \quad 1320.0$ & “ $:$ & 9.24 & " \\
\hline Cadmium . & $\ldots \ldots 13-8.7$ & $" 1 "$ & 8.85 & $"$ \\
\hline Magnesium & $\ldots \quad 1336.1$ & " " & 9.13 & $c^{i}$ \\
\hline Calcium .. & $\ldots \mathrm{I} 732 \mathrm{I}$ & $" \|$ & 7.04 & $"$ \\
\hline Strontium & $\ldots \quad 1882.3$ & “ " & 6.48 & “ \\
\hline Barium .. & $\ldots 2109.04$ & " it & 5.78 & $" 4$ \\
\hline Thallium & $\ldots \ldots 1471.74$ & “.$"$ & 8.29 & $" ،$ \\
\hline
\end{tabular}

\section{IONIZING POTENTIALS AND BOHR'S THEORY OF THE ORIGIN OF RADIATION.}

In the theory which has been brought forward by Bohr ${ }^{17}$ the atom of an element is supposed to consist of a positive Rutherford nucleus surrounded by one or more rings of electrons revolving in stationary or non-radiating orbits about the nucleus. In the neutral or most stable state the electrons are revolving in the orbits of smallest possible area. The diagram in Fig. 8 may be taken to illustrate this point.

A neutral atom may be supposed, for example, to consist of a positive nucleus $E$ surrounded by two rings of revolving electrons $A$ and $B$. If through some agency, such as an electronic bombardment, one or more of the electrons in the ring $B$ be made to revolve in the orbit $C$, then, according to the theory of Bohr, these electrons would not radiate while revolving in the orbit $C$, but they would send out a radiation of a single determinate wave-length in passing back from the orlit $C$ to the stable - orbit $B$. Extending the theory still further, if the disturbing agency caused one or more of the electrons in the orbit $B$ to revolve in the orbit $D$, then, as the electrons might drop back either directly to the orbit $B$ or to the orbit $C$ first and then to the orbit $B$, it would appear that atoms subjected to such a disturbance would be capable, in returning to the neutral state, of emitting a radia-

${ }^{17}$ Bohr, Phil. Mag., 26, pp. I, 476. 857 (1913) ; 27, p. 506 (1914); 3o, p. 394 (1915). 
tion consisting of two and possibly three definite and determinate wave-lengths. It would then seem, from Bohr's theory, that atoms of a vapor bombarded by electrons should be capable of emitting either a single-line spectrum, a two- or three-line spectrum, a four-line spectrum, etc, according to the violence of the shock to which they were subjected.

Again, according to the theory of Bohr, ionization of an atom could only be said to have taken place when the disturbing agency caused one or more of the electrons to be projected out from the electronic system beyond the outermost stationary or nonradiating orbit of the atom. This theory would, therefore,

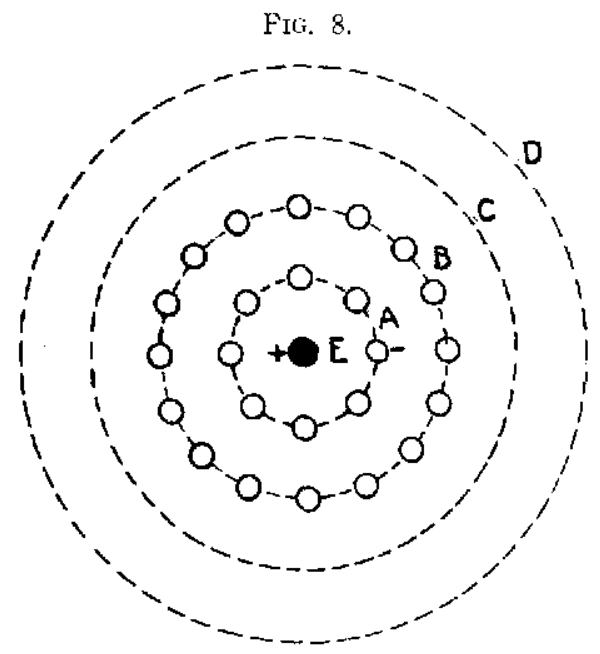

predicate but one type of ionization for atoms. By applying the theory to the matters discussed in the present communication it would appear that atoms in the state to emit a single-line spectrum could not be said to be ionized. It would follow then that if Bohr's theory of the origin of radiation be correct, the interpretation placed by Frank and Hertz on the results of their direct investigation of the ionizing potentials for mercury atoms cannot be the correct one. On the other hand, in the experiments of Henderson and myself in which the single-line spectra were obtained with mercury, zinc, and cadmium vapors when they were bombarded by electrons, the fields in which these bombarding electrons acquired their energy covered a range of from about 5 volts to slightly over ro volts. It is probable that under 
these conditions the great majority of the bombarding electrons would acquire just sufficient energy to stimulate the atoms of the vapors traversed to the emission of a radiation of but a single wave-length. The second absorption bands, however, in the absorption spectra of mercury, zinc, and cadmium vapors, it will be recalled, come at $\lambda=1849.6 \AA$. U., $\lambda=2 \mathrm{I} 39.3 \stackrel{\circ}{\Lambda}$. U., and $\lambda=$ $2288.79 \AA$. U. respectively, and it will be seen, therefore, that if the quantum relation $V e=h \nu$ be applicable the potential falls corresponding to these wave-lengths are well within the range extending from 5 to Io volts. One would have expected, therefore, that with arcing potentials of to volts one should have found traces

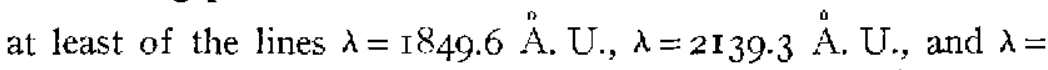
2288.79 A. U. acompanying the lines $\lambda=2536.72$ A. U., $\lambda=$ 3075.99 A. $\mathrm{L}$., and $\lambda=3260$.I7 $\AA$. U. in the spectra emitted by the three vapors. No indication of these lines, however, was found in the experiments of Henderson and myself, even with exposures of five hours' duration and with vapors covering a wide range of densities. It should be remembered that, even if some of the atoms of the vapors traversed were stimulated to the emission of the shorter wave-lengths mentioned, the radiation of these wave-lengths might have been absorbed in passing through the outer layers of the vapor in the arcing tube. The experiments of Henderson and myself cannot, therefore, be taken as being opposed to the correctness of Bohr's theory. If, however, this theory be correct, then it does follow that Frank and Hertz have incorrectly interpreted their results. Moreover, if it should turn out that they and also Newman have placed the wrong interpretation on the results of their investigations, then the ionizing potentials for morcury, zinc, cadmium, magnesium, calcium, strontium, barium, and thallium should not be those given in Table I, but they should, in all probability, be those given in Table II, and we should arrive at the conclusion that there is but one type of ionization for atoms.

\section{SUMMARY OF RESULTS.}

I. It has been show1l that, like the vapors of mercury, zinc, and cadmium, magnesium vapor, when traversed by electrons, can be stimulated to the cmission of a single-line spectrum. The wave-length of this line is given by $\lambda=2852.22 \AA$. U. 
2. It has been shown that the absorption spectrum of nonluminous magnesium vapor contains an absorption band at $\lambda=$ $2852.22 \AA$. C. and one at $\lambda=2073.36 \AA$. U.

3. As the lines $\lambda=2852.22 \AA$. U. and $\lambda=2073.36 \AA$. U. are the first members of the series $\nu=2, p_{2}-m, S$ and $\nu=1.5, S-m$, $P$ respectively, the absorption spectrum of magnesium vapor has been shown to be analogous to the absorption spectra of the vapors of mercury, zinc, and cadmium.

4. Considerations have been presented which indicate that the single-line spectra of calcium, strontium, barium, and thallium vapors under bombardment by electrons should have the wave-lengths $\lambda=4226.9 \mathrm{I} \stackrel{\AA}{\AA}$. U., $\lambda=4607.52$ A. U., $\lambda=5535.69$ $\stackrel{\circ}{\mathrm{A}} \mathrm{U}$., and $\lambda=5350.65 \AA$ А. U. respectively.

5. The ionizing potentials have been deduced for atoms of magnesium, calcium, strontium, barium, and thallium in addition to those for the atoms of mercury, zinc, and cadmium.

6. Considerations have also been presented which show that if Bohr's theory affords an explanation of the origin of singleline spectra, then Frank and Hertz and also Newman must have placed a wrong interpretation on the results of their direct investigation of the ionizing potentials for mercury atoms.

University of Toronto,

November I1, 1915 .

Turbines vs. Engines in Units of Small Capacities. J. S. BARstow. (Proceedings of the American Society of Mechanical Engineers, December 7 to IO, IO15.) - It may safely be said that not until twenty years ago was there any really reliable steam turbine developed, and even as late as ten years ago the turbine was looked upon mainly as an experiment. The last few years. however, have witnessed the practical perfection of this type of prime mover in sizes as large as 50,000 horse-power, with units of 30,000 horse-power quite common in central stations, and for installations of stch enormous output the superiority of the turbine is undisputed. In small plants of 500 horse-power or less the turbine still divides honors with the reciprocating engine, and in the present state of the art a judicious selection between them can only be made by a close analysis of all the requirements and by comparing the costs of meeting them with each type of prime mover. 